\title{
Techniques for Reducing the Complexity of Viterbi Detector
}

\author{
Muhanned AL-Rawi* \\ Department of Electrical Engineering, University of Ibb, Yemen
}

Muaayed AL-Rawi

Department of Electrical Engineering, AL-Mustansiryia University, Iraq

* Corresponding author. E-mail: muhrawi@yahoo.com

Received: 5 July 2015; Accepted: 1 October 2015; Published online: 14 October 2015

(C) 2015 King Mongkut's University of Technology North Bangkok. All Rights Reserved.

\begin{abstract}
This paper presents two detectors used to tackle intersymbol interference introduced by the communication channels. These two detectors are based on combination of nonlinear equalizer and Viterbi detector. The first detector, which was previously developed, is named Combined Detector-1(CDR1), while, the second detector, which is the contribution of this paper, is named Combined Detector-2(CDR2). These detectors are tested beside nonlinear equalizer using data transmission at $9.6 \mathrm{~kb} / \mathrm{s}$ over telephone channel. Simulation results show that the performance of CDR2 is better than the performance of CDR1 while the performance of CDR1 is better than the performance of nonlinear equalizer.
\end{abstract}

Keywords: Nonlinear equalizer, Viterbi detector, Intersymbol interference, Telephone channel, QAM modem

\section{Introduction}

In the digital data transmission system, the communication channel introduces different types of impairments, intersymbol interference (ISI) is one of those impairments. An adaptive linear or nonlinear (decision-feedback) are used to handle ISI at the receiver end [1]. It is well known that a maximum likelihood sequence estimation (MLSE), implemented with the Viterbi algorithm, can provide a significant improvement in detection performance over equalization techniques [2], [3].

When the sampled impulse response of the channel contains a large number of components, the Viterbi algorithm involves both an excessive amount of storage and an excessive number of operation per received data symbol. Considerable researches have been carried out to achieve the performance of the MLSE at reduced complexity [4]-[19].

\section{Data Transmission System}

Figure 1 shows the model of data transmission system. The first part in this model is random data generator which generates binary data, and each 4-bit is mapped into one of 16-point QAM constellation. Thus, the output of random data generator is data symbols $\left\{\mathrm{s}_{\mathrm{i}}\right\}$, and the possible values of $\mathrm{s}_{\mathrm{i}}$ are given by all combination of $\pm 1, \pm 3, \& \pm \mathrm{j} 1, \pm \mathrm{j} 3$ where $\mathrm{j}=\sqrt{-1}$. Then, the data symbols $\left\{\mathrm{s}_{\mathrm{i}}\right\}$ enter the Quadrature Amplitude Modulation (QAM) transmitter which consists of transmitter filter and QAM modulator. The transmitter filter is a low-pass filter performs the function of limiting the signal spectrum before modulation process. The resulting output of the QAM transmitter is QAM signal with carrier frequency of $1800 \mathrm{~Hz}$ and symbol rate of 2400 baud giving an information rate of $2400 \times 4 \mathrm{bits}=9600 \mathrm{~b} / \mathrm{s}=9.6 \mathrm{~kb} / \mathrm{s}$. The output of the

Please cite this article as: M. AL-Rawi and M. AL-Rawi, "Techniques for Reducing the Complexity of Viterbi Detector," KMUTNB Int J Appl Sci Technol, Vol. 8, No. 4, pp. 233-236, 2015, http://dx.doi.org/10.14416/j.ijast.2015.10.001 


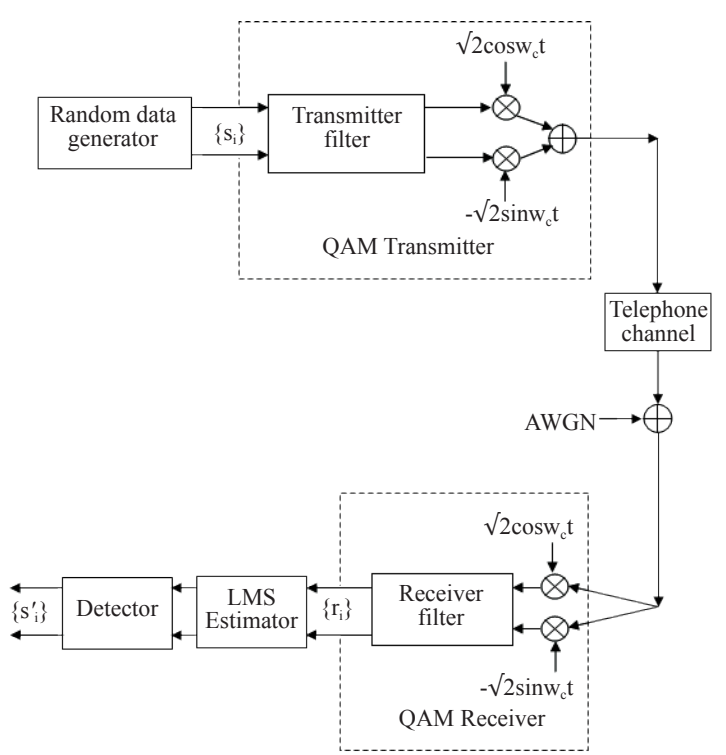

Figure 1: Model of data transmission system.

QAM transmitter passes through telephone channel, and Additive White Gaussian Noise (AWGN) added to the signal before entering the QAM receiver. The QAM receiver consists of QAM demodulator and receiver filter. The receiver filter is a low-pass filter used in combination with the transmitter filter to produce realistic levels of intersymbol interference. The output of QAM receiver is data symbols $\left\{\mathrm{r}_{\mathrm{i}}\right\}$ used by the Least Mean Square (LMS) estimator to estimate the sampled impulse response (SIR) of baseband telephone channel. Finally, the data symbols $\left\{r_{i}\right\}$ and SIR are used by the detector to obtain the detected symbols $\left\{\mathrm{s}_{\mathrm{i}}^{\prime}\right\}$.

\section{Detector Model}

\subsection{Combined Detector-1(CDR1)}

This detector, which was previously developed in [6], combines nonlinear equalizer (NLE) and Viterbi detector (VD) as shown in Figure 2. The NLE is implemented as linear feedforward transversal filter fed from the output of the VD which is the detected sample $s_{i}^{\prime}$. The output signal from this filter is subtracted from the received sample $r_{i}$ to give the input signal to VD. The NLE, therefore, operates by the quantized feedback correction, removing ISI totally or partially from the detector input signal as explained below.



Figure 2: Block diagram of CDR1.

The sampled impulse response of the baseband channel is given by $(\mathrm{g}+1)$ component row vector as

$h=\left[h_{0} h_{1} \ldots \ldots \ldots \ldots \ldots \ldots \ldots \ldots \ldots, h_{g}\right]$

Thus the received sample value is

$r_{i}=s_{i} h_{0}+\sum_{j=1}^{g} s_{i-j} h_{j}+w_{i}$

Where $S_{i}$ is the wanted transmitted sample, $w_{i}$ is noise component, and ISI is

$I S I=\sum_{j=1}^{g} s_{i-j} h_{j}$

Now, if ISI is totally removed by linear feedforward transversal filter (assuming the detected sample $s_{i}^{\prime}=$ transmitted sample $s_{i}$ ), then the input signal to VD is

$x_{i}=s_{i} h_{0}+w_{i}$

In this case, VD acts as simple threshold circuit and hence CDR1 operates as NLE. But, if part of ISI is removed by linear feedforward transversal filter, then VD tackles the remaining part of ISI, as illustrated mathematically below.

The received sample in equation (2) can be rewritten as

$r_{i}=s_{i} h_{0}+\sum_{j=1}^{m} s_{i-j} h_{j}+\sum_{j=m+1}^{g} s_{i-j} h_{j}+w_{i}$

and the input to VD is

$x_{i}=r_{i}-\sum_{j=m+1}^{g} s_{i-j} h_{j}=s_{i} h_{0}+\sum_{j=1}^{m} s_{i-j} h_{j}+w_{i}$

where the second part of ISI is removed by linear feedforward transversal filter. 


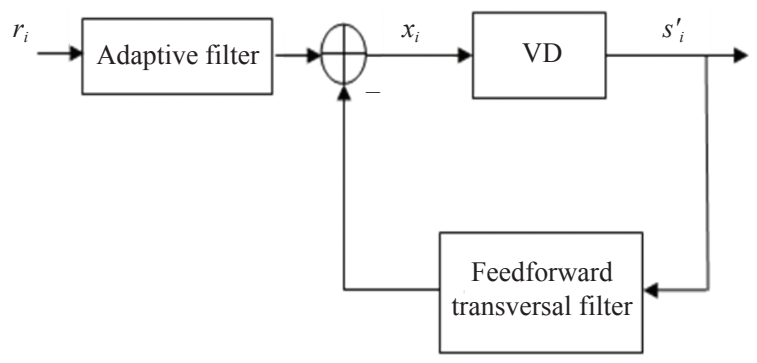

Figure 3: Block diagram of CDR2.

It is clear from equation (6), as $m$ increases, the part of ISI treated by VD increases and hence the complexity of VD increases, but the performance will improve with the increase of $m$. Here, $m$ is taken to be equal to one $(m=1)$ in order to decrease the complexity of VD, and the input to VD becomes,

$x_{i}=s_{i} h_{0}+s_{i-1} h_{1}+w_{i}$

It can be observed from equation (7) that the length of SIR of channel becomes $g+1=2$, and this reduces significantly the complexity of VD.

\subsection{Combined Detector-2(CDR2)}

In this new detector, an adaptive filter is placed at the front end of CDR1 as shown in Figure 3.

The adaptive filter is all-pass network with an infinite number of taps and will adjust the SIR of the channel and filter in cascade to be minimum phase without changing the amplitude distortion introduced by the channel and without changing the signal to noise ratio. It concentrates the energy of the channel and filter towards the earlier samples in such a way to maximize the ratio of the amplitude of the first component of SIR of the channel and filter to the output noise variance. It also removes all phase distortion introduced by the channel. The details of this filter is found in [20]. It was shown in [20] that the adaptive filter improves the performance of the detector.

Finally, it is obvious that using this filter will increase the complexity of the whole detector but at the same time will improve its performance. So, CDR2 is more complex than CDR1 but with better performance.

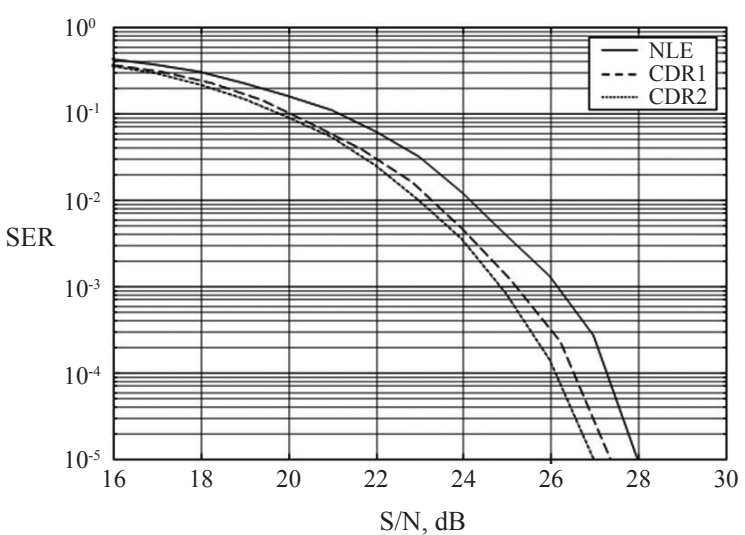

Figure 4: Error rate performance.

\section{Simulation Results}

A series of computer simulation tests have been carried out on the system in Figure 1 with three types of detectors, NLE, CDR1, and CDR2 to determine their relative tolerance to AWGN when operating over telephone channel.

The performance of the whole system is measured by drawing symbol error rate (SER) versus signal-to-noise-ratio (SNR). The SER is given by

\section{$\mathrm{SER}=\mathrm{NEDS} / \mathrm{NTS}$}

where NEDS is the number of erroneous detected samples \& NTS is the number of total transmitted samples.

Figure 4 shows the performances of the three detectors. It seems that at error rate of $10^{-5}$, the performance of CDR2 is better than the performance of CDR1 by approximately $0.4 \mathrm{~dB}$. Also, the performance of CDR1 is better than the performance of NLE by approximately $0.6 \mathrm{~dB}$.

\section{Summary and Conclusions}

Model of bandpass transmission system based on computer simulation was developed. The system operates at rate of $9.6 \mathrm{~kb} / \mathrm{s}$ using QAM signal to be transmitted over telephone channel. Three detectors have been involved in this simulation, NLE, CDR1, and CDR2. The results show that the performance of CDR1 is better than NLE but worse than CDR2. 


\section{References}

[1] A. P. Clark, Equalizers for digital modems, 1th ed. London: Pentech Press, 1985.

[2] G. Forney, "Maximum likelihood sequence estimation of digital sequences in the presence of ISI," IEEE Trans. Inf. Theory, vol. 18, no. 3, pp. 363-378, 1972.

[3] G. Forney, "The Viterbi algorithm," P. IEEE, vol. 61, no. 3, pp. 268-278, 1972.

[4] D. Falconer and F. Magee, "Adaptive channel memory truncation for maximum likelihood sequence estimation," Bell system Tech. J., vol. 52, no. 9, pp. 1541-1562, 1973.

[5] C. Beare, "The choice of the desired impulse response in combined linear-Viterbi algorithm equalizers," IEEE Trans. Commun., vol. 26, pp. 1301-1307, 1978.

[6] J. W. M. Bergmans, S. A. Rajput, and F. A. M. Van De Laar, "On the use of decision feedback for simplifying the Viterbi detector," Philips $J$. Res., vol. 42, no. 4, pp. 399-428, 1987.

[7] S. Qureshi and M. Eyubolu, "Reduced state sequence estimation with set partitioning and decision feedback," IEEE Trans. Commun., vol. 36, no. 1, pp. 13-20, 1988.

[8] A. Duel-Hallen and C. Heegard, "Delayed decision-feedback sequence estimation," IEEE Trans. Commun., vol. 37, no. 51, pp. 428-436, 1989.

[9] N. Sundstrom, O. Edfors, P. Odling, H. Eriksson, T. Koski, and P. O. Borjesson, "Combined linear-Viterbi equalizer: Comparative study and a minimax design," in Proc. 44th IEEE Vehicular Technol. Conf., 1994.

[10] R. E. Kamel and Y. Bar-Ness, "Reduced complexity sequence estimation using state partitioning," IEEE Trans. Commun., vol. 44, no. 9, pp. 1057-1063, 1996.

[11] K. Takizawa and R. Kohno, "Low complexity
Viterbi equalizer for MBOK DS-UWB systems," IEICE Trans. Fund. Electron., vol. E88-A, no. 9, 2005.

[12] T. Chien-cheng, "Symbol-based decision feedback equalizer with maximum likelihood sequence estimation for wireless receivers under multipath channels," U.S. Patent 7197094, 2007.

[13] H. Myburgh and J. Olivier, "Low complexity iterative MLSE equalization of M-QAM signals in extremely long Rayleigh fading channels," in Proc. IEEE EUROCON, Saint-Petersburg, Russia, 2009.

[14] Y. Peng, K. Zhang, A. G. Klein, and X. Huang, "Complexity and performance tradeoffs of nearoptimal detectors for cooperative ISI channels," in Proc. of IEEE Int. Conf. on Military Commun., 2010.

[15] A. Stephen and L. Quinn, "High performance equalizer having reduced complexity," U.S. Patent 7656943 B2, 2010.

[16] A. Turner-Barnes and S. Bibyk, "Is hybrid combination of Viterbi detector and decision feedback equalizer feasible in electrical SerDes?," in Proc. of DesignCon-2010, Santa Clara, California, USA, Feb. 1-4, 2010, pp. 1054.

[17] F. Rusek and A. Prlja "Optimal channel shortening for MIMO and ISI channels," IEEE Trans. Wireless Commun., vol. 11, no. 2, pp. 810-818, 2012.

[18] G. N. Maggio, M. R. Hueda, and O. E. Agazzi, "Reduced complexity MLSD receivers for nonlinear optical channels," IEEE Photon. Technol. Lett., vol. 26, no. 4, pp. 398-401, 2014.

[19] N. Zheng and T. Zhang, "Design of LowComplexity 2-D SOVA Detector for Shingled Magnetic Recording," IEEE Trans. Magn., vol. 51, no. 4, pp. 1-7, 2015.

[20] A. P Clark and S. F. Hau, "Adaptive adjustment of receiver for distorted signals," IEE Proc., vol. 131, no. 5, pp. 526-536, 1984. 